Supplement of Biogeosciences, 18, 2091-2106, 2021 https://doi.org/10.5194/bg-18-2091-2021-supplement (C) Author(s) 2021. CC BY 4.0 License.

(c) (1)

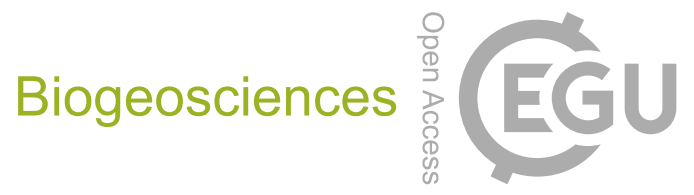

Supplement of

\title{
Metagenomic insights into the metabolism of microbial communities that mediate iron and methane cycling in Lake Kinneret iron-rich methanic sediments
}

Michal Elul et al.

Correspondence to: Michal Elul (elul@ post.bgu.ac.il)

The copyright of individual parts of the supplement might differ from the article licence. 


\section{S1. Supplementary Methods}

Method S1: Analysis of microbial diversity in slurry incubations based on the 16S rRNA gene amplicon sequencing. Total genomic DNA was extracted from duplicate samples of the slurries using the MoBio Power Soil DNA isolation kit (MoBio Laboratories, Solana Beach, CA). Genomic DNA was eluted using $80 \mu 1$ of elution buffer and stored at $-20^{\circ} \mathrm{C}$. Duplicates of $16 \mathrm{~S}$ rRNA gene fragments were joined together and amplified by PCR using a Biometra T Gradient thermocycler (Biometra, Göttingen, Germany) for MiSeq sequencing. Linkered primers that were used are 41F/806R for bacteria: (CS1-341F: 5'-ACACTGACGACATGGTTCTACACCTACGGGAGGCAGCAG, CS2806R:5'-TAC-GGTAGCAGAGACTTGGTCTGGACTACHVGGGTWTCTAAT) and Ar915- Ar1386 for archaea (CS1_Ar915F:5'-ACACTGACGACATGGTTCTACAAGGAATTGGCGGGGGAGCAC, CS2_Ar1386R: TACGGTAGCAGAGACTTGGTCTGCGGTGTGTGCAAGGAGC) for an 16S rRNA genes. All primer sets were used in PCR amplification in parallel with Dream Taq (Fermentas, Lithuania). From PCR protocol initial denaturation step of 2 min at $95^{\circ} \mathrm{C}$ was followed by 30 cycles of the following incubation pattern at $95^{\circ} \mathrm{C}$ for $20 \mathrm{~s}, 52 / 59^{\circ} \mathrm{C}$ for $20 \mathrm{~s}$ for bacteria or archaea, respectively, and $56^{\circ} \mathrm{C}$ for $65 \mathrm{~s}$. A final extension at $65^{\circ} \mathrm{C}$ for 7 min completed the reaction.

Illumina MySeq sequencing of the PCR products was performed at DNA Services (DNAS) Facility (Research Resources Center University of Illinois at Chicago). Demultiplexed paired-end reads were analyzed using QIIME2 V2019.7 (Rideout et al. 2018). Reads were truncated based on quality plots, checked for chimeras, merged and grouped into amplicon sequence variants (ASVs) with DADA2 (Callahan et al. 2016), as implemented in QIIME2. A NaïveBayes classifier trained on the Silva 132 full 99\%-clustered 16S rRNA sequences. Representative sequences were aligned with MAFFT (Katoh and Standley 2013), masked, and trees were generated using FastTree (Price et al. 2009), as implemented in QIIME2. Downstream statistical analyses and plotting were performed in R (R Core Team 2018), using libraries phyloseq (McMurdie and Holmes 2013), ampvis2 (Andersen et al. 2018) and ggplot2 (Wickham 2009). 


\section{S2. Supplementary Figures}

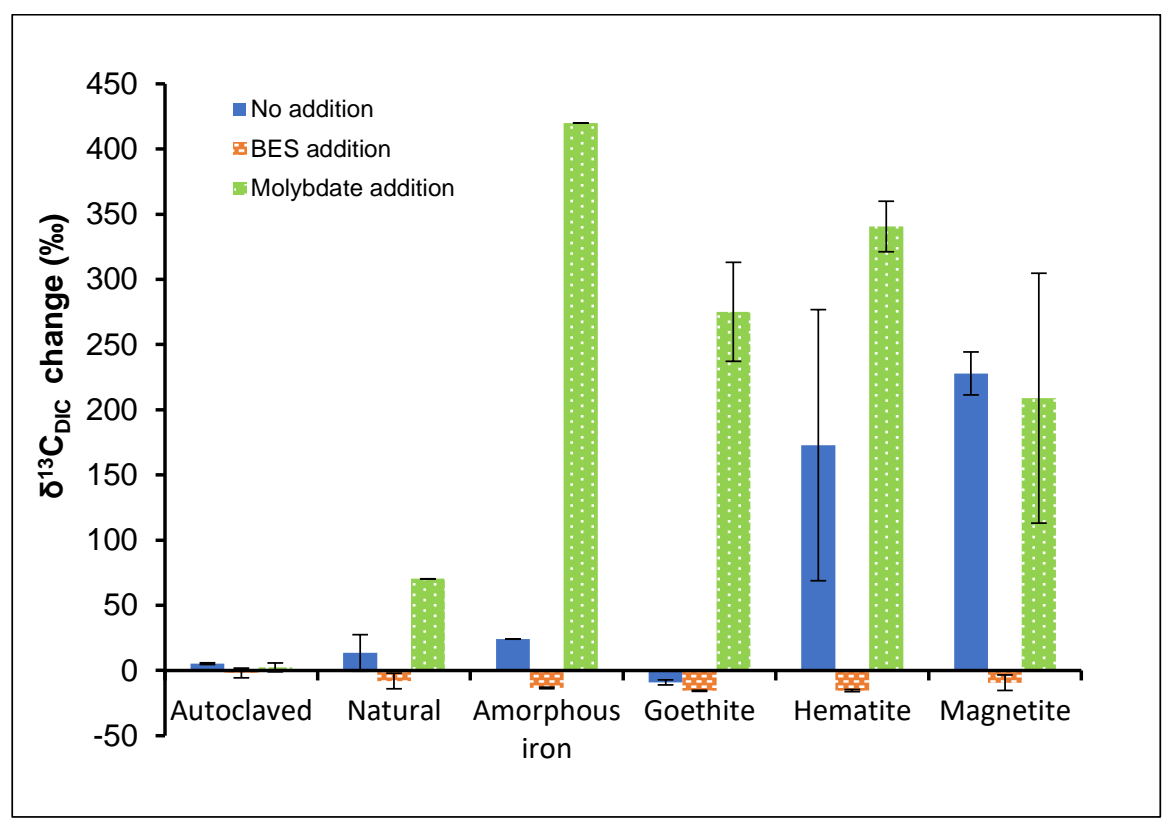

Figure S1: Net change in $\delta^{13} \mathrm{C}_{\text {DIC }}$ values in Bar-or et al.2017 slurry incubations after 470 days. Solid blue: without inhibitor addition; Fence red: inhibition of methanogenesis by BES addition; Dot green: inhibition of sulfate reduction by molybdate addition. Analysis of DNA 16S rRNA genes was performed for all of these incubations and the untreated sediments. The following treatments: Natural (without additions), Amorphous iron with the addition of molybdate and the hematite (without additions) treatment were sequenced for metagenome analysis. After Bar-Or et al. 2017 
Proteobacteria; Gammaproteobacteria; Enterobacteriales es; Bacilli; Bacillales

cteria; Pseudomonadales Firmicutes; Clostridia; Clostridiales

Proteobacteria, Gammaproteobacteria; Methylococcales Proteobacteria, Deltaproteobacteria;

Acidobacteria; Aminicenantia; Aminicenantales

Chloroflexi; Dehalococcoidia; GIFg

acteroidetes; Bacteroidia; Bacteroidales

Chescribar

Omnitrophicaeota order

Chloroflexi; SHA-26 order

Proteobacteria; Deltaproteobacteria order

Chloroflexi; Dehalococcoidia; FS117-23B-02

Proteobacteria; Gammaproteobacteria; Acidiferrobacterales

Rokubacteria; NC10; Methylomirabilales

Proteobacteria; Deltaproteobacteria; Desulfarculales

Proteobacteria; Deltaproteobacteria; MBNT15

Proteobacteria; Gammaproteobacteria; HOC36

Patescibacteria; Patescibacteria; Patescibacteria

Preocacteria, Deltaproteobacteria, Desulfobacterales

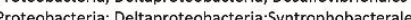

Bacteroidetes; Ignavibacteria; Kryptoniales

WOR-1 order

Chloroflexi; Anaerolineae order

Calditrichaeota; Calditrichia; Calditrichales

Esilonbacteraeota; Campylobacteria; Campylobacterales

irmicutes; Negativicutes; Selenomonadales

Nitrospirae; Thermodesulfovibrionia order

(

Proteriacter

Nitrospirae; Thermodesulfovibrionia orde

Chloroflexi; Anaerolineae; Anaerolineales

roteobacteriales

Bacteroidetes; Bacteroidia; Bacteroidales

Acidobacteria; Aminicenantia; Aminicenantales

Chorolexi, Dehalococcolda, MSBL5

Proteobacteria; Deltaproteobacteria; Sva0485

Proteobacteria; Gammaproteobacteria; Methylococcales

Proteobacteria; Deltaproteobacteria; Desulfuromonadales

Chloroflexi; SHA-26 order

Calditrichaeota; Calditrichia; Calditrichales

Omnitrophicaeota order

Proteobacteria; Deltaproteobacteria order

Chloroflexi; Dehalococcoidia; FS117-23B-02

Proteobacteria; Gammaproteobacteria; Aci

Rokubacteria; NC10; Methylomirabilales

Proteobacteria; Deltaproteobacteria; Desulfarculales

Prescibacteria, Parcubacteria; Parcubacteria

Proter

Chloroflexi order

Patescibacteria order

Patescibacteria; Parcubacteria order

Chloroflexi; Anaerolineae order

Proteobacteria; Deltaproteobacteria; Desulfobacterales Proteobacteria; Deltaproteobacteria; Desulfovibrionales

Chloroflexi; Anaerolineae Order

acteroidetes; Ignavibacteria; Kryptoniales

Proteobacteria; Deltaproteobacteria; Syntrophobacterales WOR-1 order

Proteobacteria; Deltaproteobacteria; Myхococcales

Prom
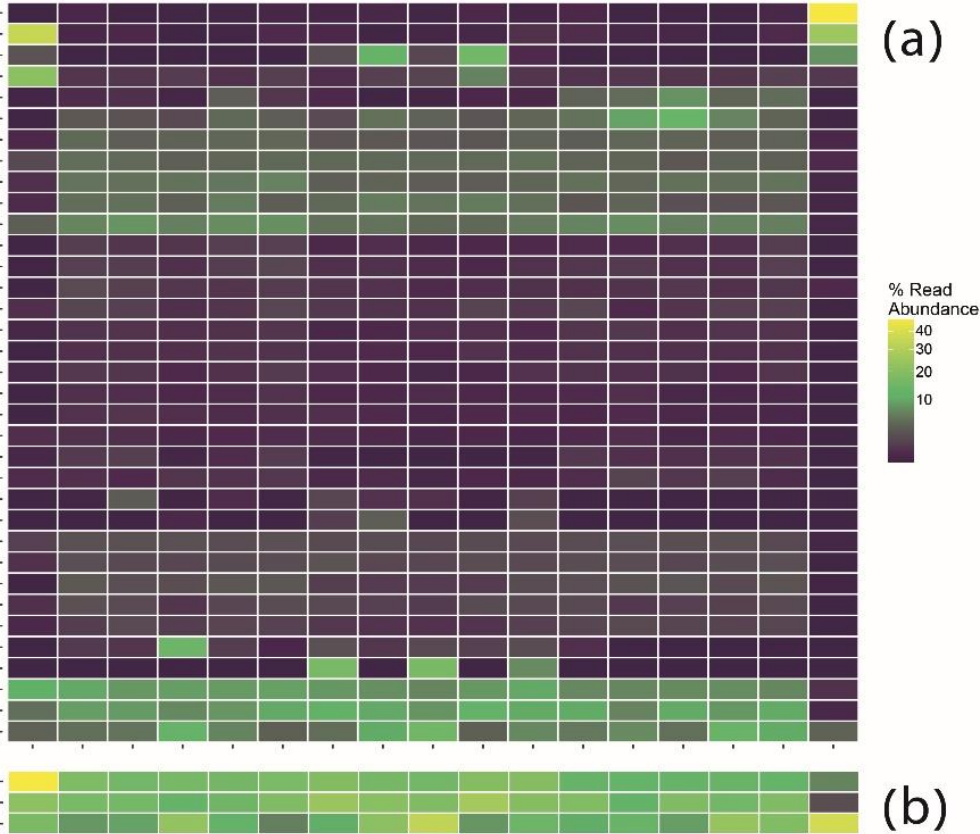

(b)

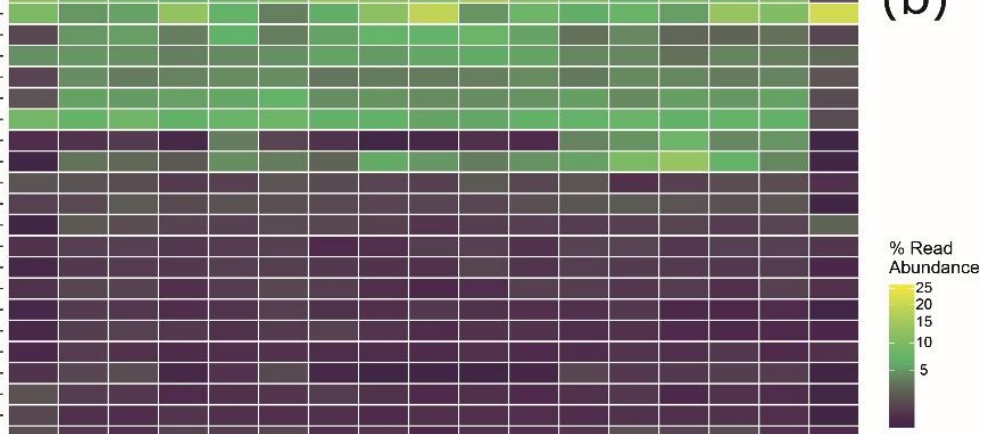

Figure S2: The relative abundance of bacteria (top 35) at the order level in Bar-Or et al. 2017 study, based on amplicon sequencing of the 16S rRNA genes, including (a) and excluding (b) the major contaminants. 
Nanoarchaeaeota; Woesearchaeia order

Euryarchaeota; hermoplasmata; MBGD and DHVEG-1

Euryarchaeota; Methanomicrobia; Methanosarcinales

Nanoarchaeaeota; Nanohaloarchaeia; DSEG

Euryarchaeota; Thermoplasmata order

Euryarchaeota; Methanomicrobia; ANME-1

Euryarchaeota; Thermoplasmata; Methanomassiliicoccale

Euryarchaeota; Thermococci; Methanofastidiosales

Crenarchaeota; Bathyarchaeia; order

Euryarchaeota; Methanomicrobia; Methanomicrobiales

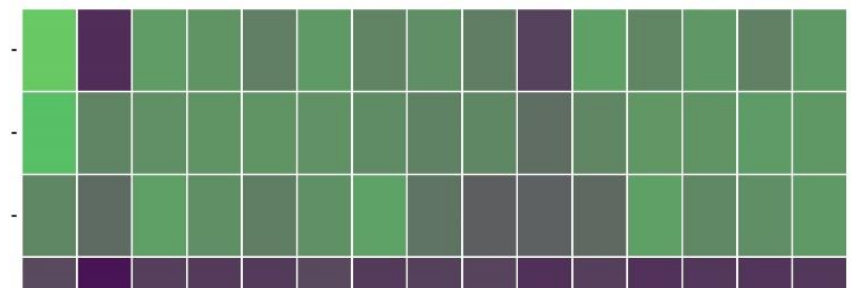

\section{$\%$ Read} Abundance

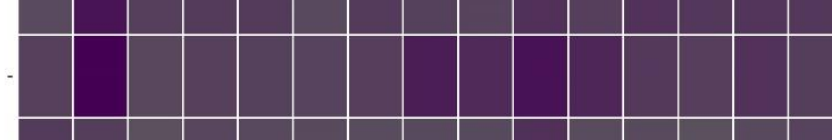

50
40
30
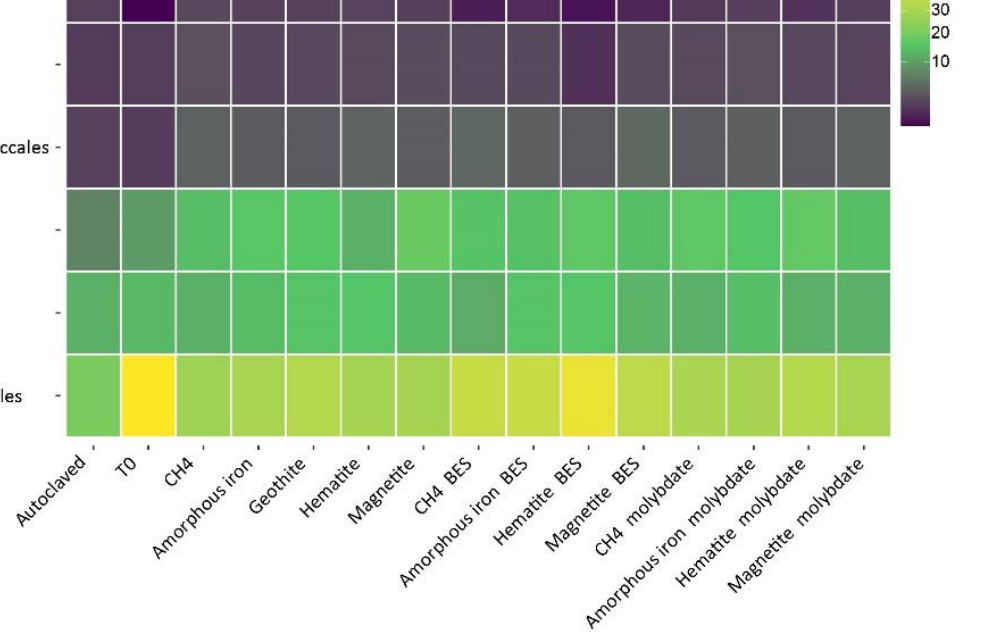

Figure S3: The relative abundance of Archaea (top 10) at the order level in Bar-Or et al. 2017 study based on amplicon sequencing of the 16S rRNA genes. 
Acidobacteria; Aminicenantia; Aminicenantales Acidobacteria; Thermoanaerobaculia; Thermoanaerobaculales Actinobacteria; Coriobacteriia; OPB41

Bacteroidetes; Bacteroidia; Bacteroidales

Calditrichaeota; Calditrichia; Calditrichales

Chloroflexi; Anaerolineae; Anaerolineales

Chloroflexi; Anaerolineae;SJA-15

Chloroflexi; Anaerolineae order

Chloroflexi; Dehalococcoidia; GIF9

Chloroflexi; Dehalococcoidia; MSBL5

Crenarchaeota; Bathyarchaeia order1

Crenarchaeota; Bathyarchaeia order2

Euryarchaeota ;Methanomicrobia; Methanomicrobiales

Euryarchaeota; Methanomicrobia; Methanosarcinales

Euryarchaeota; Thermococci; Methanofastidiosales

Euryarchaeota; Thermoplasmata; MBGD and DHVEG-1

Euryarchaeota; Thermoplasmata; Methanomassiliicoccales

Firmicutes; Bacilli; Bacillales

Firmicutes; Bacilli; Lactobacillales

Firmicutes; Clostridia; Clostridiales

Kiritimatiellaeota; Kiritimatiellae; WCHB1-41

Nanoarchaeaeota; Woesearchaeia order1

Nanoarchaeaeota; Woesearchaeia order2

Nitrospirae; Thermodesulfovibrionia order

Omnitrophicaeota class

Patescibacteria; Microgenomatia; Candidatus Woesebacteria

Planctomycetes; Phycisphaerae; MSBL9

Planctomycetes; Planctomycetacia; Pirellulales

Proteobacteria; Deltaproteobacteria; Desulfarculales

Proteobacteria; Deltaproteobacteria; Desulfuromonadales

Proteobacteria; Deltaproteobacteria; MBNT15

Proteobacteria; Deltaproteobacteria; Myxococcales

Proteobacteria; Deltaproteobacteria; Sva0485

Proteobacteria; Deltaproteobacteria; Syntrophobacterales

Proteobacteria; Gammaproteobacteria; Betaproteobacteriales -

Proteobacteria; Gammaproteobacteria; HOC36

Proteobacteria; Gammaproteobacteria; Methylococcales

Proteobacteria; Gammaproteobacteria; Steroidobacterales

Spirochaetes; Spirochaetia; Spirochaetales

\begin{tabular}{|c|c|c|c|c|}
\hline 0 & 0 & 0 & 0 & 0 \\
\hline 0 & 0 & 0 & 0 & 0 \\
\hline • & $\circ$ & 0 & $\circ$ & $\circ$ \\
\hline 0 & 0 & 0 & $\circ$ & 0 \\
\hline 0 & 0 & 0 & 0 & 0 \\
\hline 0 & 0 & 0 & 0 & 0 \\
\hline$\bullet$ & $\circ$ & 0 & $\circ$ & 0 \\
\hline 0 & 0 & 0 & 0 & 0 \\
\hline 0 & 0 & 0 & 0 & 0 \\
\hline 0 & 0 & 0 & 0 & 0 \\
\hline 0 & 0 & 0 & 0 & 0 \\
\hline ○ & 0 & 0 & 0 & 0 \\
\hline 0 & 0 & 0 & 0 & 0 \\
\hline 0 & 0 & 0 & ○ & 0 \\
\hline 0 & 0 & 0 & 0 & 0 \\
\hline 0 & 0 & 0 & ० & 0 \\
\hline 0 & 0 & $\circ$ & , & 0 \\
\hline • & 0 & $\circ$ & & 0 \\
\hline & $\circ$ & - & 0 & $\circ$ \\
\hline$\bullet$ & $\circ$ & $\circ$ & 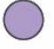 & 0 \\
\hline$\bullet$ & 0 & 0 & $\circ$ & 0 \\
\hline 0 & 0 & $\circ$ & $\circ$ & 0 \\
\hline 0 & 0 & $\circ$ & 0 & 0 \\
\hline 0 & 0 & 0 & 0 & 0 \\
\hline 0 & 0 & 0 & 0 & 0 \\
\hline 0 & 0 & 0 & 0 & 0 \\
\hline 0 & 0 & 0 & 0 & 0 \\
\hline • & 0 & 0 & 0 & 0 \\
\hline 0 & 0 & 0 & 0 & 0 \\
\hline 0 & 0 & 0 & ○ & 0 \\
\hline 0 & 0 & 0 & 0 & 0 \\
\hline 0 & 0 & 0 & 0 & 0 \\
\hline 0 & 0 & 0 & 0 & 0 \\
\hline 0 & 0 & 0 & 0 & 0 \\
\hline 0 & 0 & 0 & 0 & 0 \\
\hline 0 & 0 & 0 & 0 & 0 \\
\hline 0 & 0 & ० & $\circ$ & 0 \\
\hline 0 & $\circ$ & 0 & 0 & 0 \\
\hline 0 & 0 & 0 & 0 & 0 \\
\hline
\end{tabular}

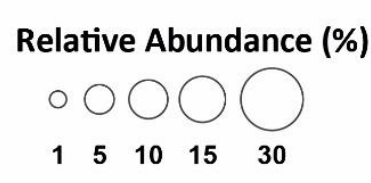

Figure S4: Relative abundance of Bacteria (black) and Archaea (green) at the order level in all five metagenomics libraries based on the mapping of metagenomic reads to the Silva132 database of the small subunit rRNA sequences. Contamination of common laboratory bacteria, such as Firmicutes and Clostridia are seen in sample t0-2013. Lineages < $1 \%$, which account together for $20-32 \%$ of the microbial community, were removed from the display. 


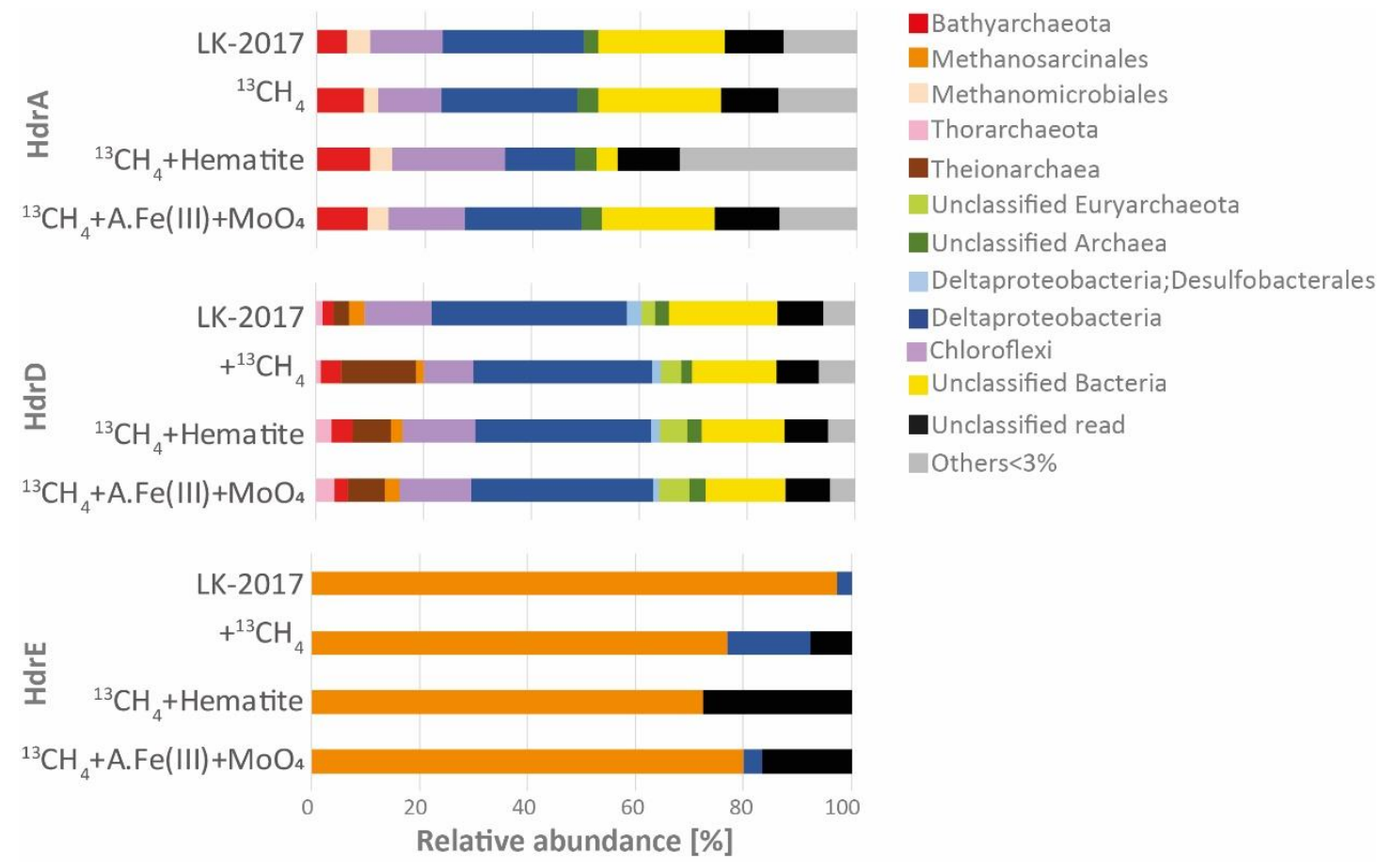

Figure S5: Phylogenetic diversity of HdrA, D and E subunits of the heterodisulfide reductase. Phylogenetic assignments are based on BLAST mapping against the RefSeq database. Taxonomic classifications at the highest level possible (up to the Order level) are shown. A.Fe(III)+Mo= amorphous iron and molybdate. 


\section{S3. Supplementary Tables}

Table S1: The various treatments in all slurry incubations, from Bar-Or et al. 2017.

\begin{tabular}{|c|c|c|c|c|c|c|}
\hline Slurry label & $\begin{array}{l}\text { amorphous } \\
\text { iron }(0.1 g)\end{array}$ & $\begin{array}{c}\text { goethite } \\
(0.1 g)\end{array}$ & $\begin{array}{c}\text { hematite } \\
(0.1 g)\end{array}$ & $\begin{array}{l}\text { magnetite } \\
(0.1 g)\end{array}$ & $\begin{array}{l}\text { BES } \\
(0.5 \mathrm{ml})\end{array}$ & $\begin{array}{c}\text { molybdate } \\
(0.5 \mathrm{ml})\end{array}$ \\
\hline $\begin{array}{l}\text { Autoclaved after: }{ }^{13} \mathrm{CH}_{4}+\text { all iron } \\
\text { minerals }\end{array}$ & $\begin{array}{l}X \\
X\end{array}$ & $\begin{array}{l}X \\
X\end{array}$ & $\begin{array}{l}X \\
X\end{array}$ & $\begin{array}{l}X \\
X\end{array}$ & & \\
\hline $\begin{array}{l}\text { Autoclaved after: }{ }^{13} \mathrm{CH}_{4}+\text { all iron } \\
\text { minerals+BES }\end{array}$ & $\begin{array}{l}\mathrm{X} \\
\mathrm{X}\end{array}$ & $\begin{array}{l}\mathrm{X} \\
\mathrm{X}\end{array}$ & $\begin{array}{l}X \\
X\end{array}$ & $\begin{array}{l}X \\
X\end{array}$ & $\begin{array}{l}\mathrm{X} \\
\mathrm{X}\end{array}$ & \\
\hline $\begin{array}{l}\text { Autoclaved after: }{ }^{13} \mathrm{CH}_{4}+\text { all iron } \\
\text { minerals+molybdate }\end{array}$ & $\begin{array}{l}X \\
X\end{array}$ & $\begin{array}{l}X \\
X\end{array}$ & $\begin{array}{l}X \\
X\end{array}$ & $\begin{array}{l}X \\
X\end{array}$ & & $\begin{array}{l}X \\
X\end{array}$ \\
\hline${ }^{13} \mathrm{CH}_{4}$ & & & & & & \\
\hline${ }^{13} \mathrm{CH}_{4}+\mathrm{BES}$ & & & & & $\begin{array}{l}\mathrm{X} \\
\mathrm{X}\end{array}$ & \\
\hline${ }^{13} \mathrm{CH}_{4}+$ amorphous iron & $\begin{array}{l}\mathrm{X} \\
\mathrm{X}\end{array}$ & & & & & \\
\hline${ }^{13} \mathrm{CH}_{4}+$ amorphous iron+BES & $\begin{array}{l}\mathrm{X} \\
\mathrm{X}\end{array}$ & & & & $\begin{array}{l}\mathrm{X} \\
\mathrm{X}\end{array}$ & \\
\hline${ }^{13} \mathrm{CH}_{4}+$ goethite & & $\begin{array}{l}X \\
X\end{array}$ & & & & \\
\hline${ }^{13} \mathrm{CH}_{4}+$ goethite+BES & & $\begin{array}{l}X \\
X\end{array}$ & & & $\begin{array}{l}X \\
X\end{array}$ & \\
\hline${ }^{13} \mathrm{CH}_{4}+$ hematite & & & $\begin{array}{l}X \\
X\end{array}$ & & & \\
\hline${ }^{13} \mathrm{CH}_{4}+$ hematite+BES & & & $\begin{array}{l}X \\
X\end{array}$ & & $\begin{array}{l}X \\
X\end{array}$ & \\
\hline${ }^{13} \mathrm{CH}_{4}+$ magnetite & & & & $\begin{array}{l}X \\
X\end{array}$ & & \\
\hline${ }^{13} \mathrm{CH}_{4}+$ magnetite+BES & & & & $\begin{array}{l}X \\
X\end{array}$ & $\begin{array}{l}X \\
X\end{array}$ & \\
\hline${ }^{13} \mathrm{CH}_{4}+$ molybdate & & & & & & $\begin{array}{l}\mathrm{X} \\
\mathrm{X}\end{array}$ \\
\hline $\begin{array}{l}{ }^{13} \mathrm{CH}_{4}+\text { amorphous } \\
\text { iron+molybdate }\end{array}$ & $\begin{array}{l}X \\
X\end{array}$ & & & & & $\begin{array}{l}X \\
X\end{array}$ \\
\hline${ }^{13} \mathrm{CH}_{4}+$ goethite+molybdate & & $\begin{array}{l}X \\
X\end{array}$ & & & & $\begin{array}{l}X \\
X\end{array}$ \\
\hline${ }^{13} \mathrm{CH}_{4}+$-hematite+molybdate & & & $\begin{array}{l}\mathrm{X} \\
\mathrm{X}\end{array}$ & & & $\begin{array}{l}X \\
X\end{array}$ \\
\hline${ }^{13} \mathrm{CH}_{4}+$ magnetite+molybdate & & & & $\begin{array}{l}X \\
X\end{array}$ & & $\begin{array}{l}X \\
X\end{array}$ \\
\hline
\end{tabular}


Table S2: Thermodynamic calculation for the feasibility of active Fe-AOM in Lake Kinneret methanic zone:

Fe-AOM

Calculation of $\Delta \mathbf{G}$ standard state

\begin{tabular}{|c|c|c|c|c|}
\hline \multicolumn{5}{|c|}{$\mathrm{CH}_{4}+8 \mathrm{Fe}(\mathrm{OH})_{3}+15 \mathrm{H}^{+} \rightarrow \mathrm{HCO}_{3}{ }^{-}+8 \mathrm{Fe}^{2+}+21 \mathrm{H}_{2} \mathrm{O}$} \\
\hline Exponent & Species name & Chemical & state & $\Delta \mathrm{G} 0$ \\
\hline 8 & Ferrous ion & $\mathrm{Fe}_{2}{ }^{+}$ & aq & -78.9 \\
\hline 21 & Water & $\mathrm{H}_{2} \mathrm{O}$ & 1 & -237.2 \\
\hline 1 & Bicarbonate & $\mathrm{HCO}_{3}{ }^{-}$ & aq & -586.9 \\
\hline 8 & Ferric-hydroxide prec. & $\mathrm{Fe}(\mathrm{OH})_{3}$ & s & -696.6 \\
\hline 15 & Proton & $\mathrm{H}^{+}$ & aq & 0 \\
\hline 1 & Methane & $\mathrm{CH}_{4}$ & aq & -34.4 \\
\hline
\end{tabular}

$\Delta \mathbf{G 0}$

$-592.1 \mathrm{~kJ} \mathrm{~mol} \mathbf{~}^{-1}$

\begin{tabular}{lrl} 
Fe-AOM & & \\
\hline G0 & -592.1 & $\mathrm{~kJ} \mathrm{~mol}^{-1}$ \\
$\mathrm{~T}$ & 293 & $\mathrm{~K}$ \\
$\mathrm{R}$ & 0.0083 & $\mathrm{KJ} /\left(\mathrm{mol}^{*} \mathrm{~K}\right)$ \\
$\mathrm{Q}$ & $7.81 \times 10^{79}$ & \\
$\operatorname{lnQ}$ & 183.96 & \\
RTlnQ & 448.15 & $\mathrm{~kJ} \mathrm{~mol}^{-1}$ \\
$\boldsymbol{\Delta} \mathbf{G}$ & $\mathbf{- 1 4 4}$ & $\mathbf{~ k J ~ m o l}^{-1}$
\end{tabular}

Acetate oxidation by ferrous iron reduction

Calculation of $\Delta \mathbf{G}$ standard state

\begin{tabular}{|c|c|c|c|c|}
\hline \multicolumn{2}{|c|}{$\mathrm{Rx}: \mathrm{CH}_{3} \mathrm{COOH}+8 \mathrm{Fe}(\mathrm{OH})_{3}+14 \mathrm{H}^{+} \rightarrow 8 \mathrm{Fe}^{2+}+2 \mathrm{HCO}_{3}+20 \mathrm{H}_{2} \mathrm{O}$} \\
\hline Exponent & Species name & Chemical & state & $\Delta \mathrm{G} 0$ \\
\hline 8 & Ferrous ion & $\mathrm{Fe}^{2+}$ & aq & -78.9 \\
\hline 20 & Water & $\mathrm{H}_{2} \mathrm{O}$ & 1 & -237.2 \\
\hline 2 & Bicarbonate & $\mathrm{HCO}_{3}^{-}$ & $\mathrm{aq}$ & -586.9 \\
\hline 8 & Ferric-hydroxide prec. & $\mathrm{Fe}(\mathrm{OH})_{3}$ & s & -696.6 \\
\hline 14 & Proton & $\mathrm{H}^{+}$ & $\mathrm{aq}$ & 0 \\
\hline 1 & Acetic acid & $\mathrm{CH}_{3} \mathrm{COOH}$ & $\mathrm{aq}$ & -396.6 \\
\hline
\end{tabular}

$\Delta G 0$

$-579.6 \mathrm{~kJ} \mathrm{~mol}^{-1}$

Acetate oxidation by ferrous iron reduction

\begin{tabular}{|c|c|c|}
\hline G0 & -579.6 & $\mathrm{~kJ} \mathrm{~mol}^{-1}$ \\
\hline $\mathrm{T}$ & 293 & $\mathrm{~K}$ \\
\hline $\mathrm{R}$ & 0.0083 & $\mathrm{KJ} /\left(\mathrm{mol}^{*} \mathrm{~K}\right)$ \\
\hline $\mathrm{Q}$ & $3.91 \times 10^{72}$ & \\
\hline $\ln Q$ & 167.15 & \\
\hline $\mathrm{RT} \ln \mathrm{Q}$ & 407.2 & $\mathrm{~kJ} \mathrm{~mol}^{-1}$ \\
\hline$\Delta \mathbf{G}$ & -172 & $\mathbf{k J} \mathrm{mol}^{-1}$ \\
\hline
\end{tabular}

$\mathrm{H}_{2}$ Iron reduction

Calculation of $\boldsymbol{\Delta} \mathbf{G}$ standard state

\begin{tabular}{|c|c|c|c|c|}
\hline \multicolumn{6}{|c|}{$8 \mathrm{Fe}(\mathrm{OH})_{3}+4 \mathrm{H}_{2}+16 \mathrm{H}^{+} \rightarrow 8 \mathrm{Fe}^{2+}+24 \mathrm{H}_{2} \mathrm{O}$} \\
\hline Exponent & Species name & Chemical & state & $\Delta \mathrm{G} 0$ \\
\hline 24 & Water & $\mathrm{H} 2 \mathrm{O}$ & 1 & -237.2 \\
\hline 8 & Ferrous ion & $\mathrm{Fe} 2+$ & aq & -78.9 \\
\hline 8 & Ferric-hydroxide prec. & $\mathrm{Fe}(\mathrm{OH}) 3$ & $\mathrm{~s}$ & -696.6 \\
\hline 4 & Hydrogen & $\mathrm{H} 2$ & aq & 17.55 \\
\hline 16 & Proton & $\mathrm{H}+$ & aq & 0 \\
\hline
\end{tabular}

$\Delta G 0 \quad-821.4 \quad \mathrm{~kJ} 4 \mathrm{~mol} \mathrm{H}^{-1}$

Acetoclastic methanogenesis

Calculation of $\Delta \mathbf{G}$ standard state

\begin{tabular}{|c|c|c|c|c|}
\hline \multicolumn{5}{|c|}{$\mathrm{CH}_{3} \mathrm{COOH} \rightarrow \mathrm{CH}_{4}+\mathrm{CO}_{2}$} \\
\hline Exponent & Species name & Chemical & state & $\Delta \mathrm{G} 0$ \\
\hline 1 & Methane & $\mathrm{CH}_{4}$ & aq & -34.4 \\
\hline 1 & Carbon dioxide & $\mathrm{CO}_{2}$ & aq & -386 \\
\hline 1 & Acetic acid & $\mathrm{CH}_{3} \mathrm{COOH}$ & aq & -396.6 \\
\hline
\end{tabular}

$\Delta \mathbf{G 0}$

$-23.8 \mathrm{~kJ} \mathrm{~mol}{ }^{-1}$

Acetoclastic methanogenesis

\begin{tabular}{lrl}
\hline G0 & -23.8 & $\mathrm{~kJ} \mathrm{~mol}^{-1}$ \\
T & 293 & $\mathrm{~K}$ \\
$\mathrm{R}$ & 0.0083 & $\mathrm{KJ} /\left(\mathrm{mol}^{* \mathrm{~K}}\right)$ \\
$\mathrm{Q}$ & 0.5 & \\
$\ln \mathrm{Q}$ & -0.69 & \\
$\mathrm{RT} \ln \mathrm{Q}$ & -1.69 & $\mathrm{~kJ} \mathrm{~mol}-1$ \\
$\mathbf{\Delta G}$ & $\mathbf{- 2 5}$ & $\mathbf{~ k J ~ m o l}^{-1}$
\end{tabular}

\begin{tabular}{lrl} 
H2 iron reduction & & \\
\hline $\mathrm{G} 0$ & -821.4 & $\mathrm{~kJ} \mathrm{mol^{-1 }}$ \\
$\mathrm{T}$ & 293 & $\mathrm{~K}$ \\
$\mathrm{R}$ & 0.0083 & $\mathrm{KJ} /\left(\mathrm{mol}^{*} \mathrm{~K}\right)$ \\
$\mathrm{Q}$ & $3.91 \times 10^{105}$ & \\
$\mathrm{lnQ}$ & 243.13 & \\
$\mathrm{RT} \operatorname{lnQ}$ & 592.31 & $\mathrm{~kJ} \mathrm{mol^{-1 }}$ \\
$\mathbf{\Delta G}$ & $\mathbf{- 2 2 9}$ & $\mathbf{k J ~ 4} \mathbf{m o l ~ H}^{-\mathbf{1}}$
\end{tabular}


$\mathrm{H}_{2}$ methanogenesis

Calculation of $\Delta \mathbf{G}$ standard state

\begin{tabular}{|c|c|c|c|c|}
\hline \multicolumn{5}{|c|}{$\mathrm{HCO}_{3}{ }^{-}+4 \mathrm{H}_{2}+\mathrm{H}^{+} \rightarrow \mathrm{CH}_{4}+3 \mathrm{H}_{2} \mathrm{O}$} \\
\hline Exponent & Species name & Chemical & state & $\Delta \mathrm{G} 0$ \\
\hline 1 & Methane & $\mathrm{CH}_{4}$ & aq & -34.4 \\
\hline 3 & Water & $\mathrm{H}_{2} \mathrm{O}$ & 1 & -237.2 \\
\hline 1 & Bicarbonate & $\mathrm{HCO}_{3}{ }^{-}$ & aq & -586.9 \\
\hline 4 & Hydrogen & $\mathrm{H}_{2}$ & aq & 17.55 \\
\hline 1 & Proton & $\mathrm{H}^{+}$ & aq & 0 \\
\hline
\end{tabular}

$\Delta \mathbf{G 0}$

$-229.3 \mathrm{~kJ} 4 \mathrm{~mol} \mathrm{H}_{2}^{-1}$

\section{$\mathrm{H}_{2}$ methanogenesis}

Calculation of dG standard state

\begin{tabular}{|c|c|c|c|c|}
\hline \multicolumn{6}{|c|}{$\mathbf{C} 2-+\mathbf{4 H}$} & $\mathbf{C H} 4+\mathbf{2} \mathbf{H}_{2} \mathbf{O}$ \\
\hline Exponent & Species name & Chemical & state & $\Delta \mathrm{G} 0$ \\
\hline 1 & Methane & $\mathrm{CH} 4$ & $\mathrm{aq}$ & -34.4 \\
\hline 2 & Water & $\mathrm{H} 2 \mathrm{O}$ & 1 & -237.2 \\
\hline 1 & Carbon dioxide & $\mathrm{CO} 2$ & $\mathrm{aq}$ & -386 \\
\hline 4 & Hydrogen & $\mathrm{H} 2$ & $\mathrm{aq}$ & 17.55 \\
\hline
\end{tabular}

H2 methanogenesis

$\begin{array}{lrl}\text { G0 } & -229.3 & \mathrm{~kJ} \mathrm{~mol}^{-1} \\ \mathrm{~T} & 293 & \mathrm{~K} \\ \mathrm{R} & 0.0083 & \mathrm{KJ} /\left(\mathrm{mol}^{*} \mathrm{~K}\right) \\ \mathrm{Q} & 5 \times 10^{31} & \\ \ln \mathrm{Q} & 72.99 & \\ \mathrm{RT} \operatorname{lnQ} & 177.81 & \mathrm{~kJ} \mathrm{\textrm {mol } ^ { - 1 }} \\ \mathbf{\Delta G} & \mathbf{- 5 1} & \mathbf{k J ~ 4} \mathbf{m o l ~ H}_{\mathbf{2}}^{-\mathbf{1}}\end{array}$

$\Delta G 0 \quad-193.0 \mathrm{~kJ} 4 \mathrm{~mol} \mathrm{H}^{-1}$

\begin{tabular}{|l|rl|}
\hline $\mathrm{H}_{2}$ meth +Fe-AOM & -195 & $\mathrm{~kJ} \mathrm{~mol}^{-1}$ \\
$\mathrm{H}_{2}$ Fe red & -229 & $\mathrm{~kJ} \mathrm{~mol}^{-1}$ \\
$\mathrm{Ac}$ meth + Fe-AOM & -169 & $\mathrm{~kJ} \mathrm{~mol}^{-1}$ \\
$\mathrm{Ac}$ Fe red & -172 & $\mathrm{~kJ} \mathrm{~mol}^{-1}$ \\
\hline
\end{tabular}

\begin{tabular}{|l|c|c|c|}
\hline \multicolumn{4}{|c|}{ Concentrations in the sediment (After Sivan et al., 2011; Adler, 2016) } \\
\hline & & $\mathrm{mM}$ & $\mathrm{M}$ \\
\hline DIC conc. Used for $\mathrm{CO}_{2}$ & $\mathrm{HCO}^{-}$ & 10 & 0.01 \\
\hline Measured & $\mathrm{Fe}^{2+}$ & 0.1 & 0.0001 \\
\hline & $\mathrm{CH}_{4}$ & 0.5 & 0.0005 \\
\hline & $\mathrm{reac} \mathrm{Fe}(\mathrm{III})$ & 200.0 & 0.2 \\
\hline $\mathrm{pH}=7$ & $\mathrm{H}_{+}$ & $1.00 \mathrm{E}-04$ & $1.00 \mathrm{E}-07$ \\
\hline & $\mathrm{H}_{2}$ & 0.01 & 0.00001 \\
\hline Measured & acetate & 0.01 & 0.00001 \\
\hline & water & 1 & 1 \\
\hline
\end{tabular}

\begin{tabular}{|ll|c|c|}
\hline \multirow{2}{*}{$\Delta$ G standard state } & & \multicolumn{2}{|c|}{} \\
\hline Hydrogen & $\mathrm{H}_{2}$ & $\mathrm{aq}$ & $17.55 \mathrm{~kJ} \mathrm{~mol}^{-1}$ \\
\cline { 3 - 4 } Ferric-hydroxide prec. & $\mathrm{Fe}(\mathrm{OH})_{3}$ & $\mathrm{~s}$ & $-696.6 \mathrm{~kJ} \mathrm{~mol}^{-1}$ \\
\cline { 3 - 4 } Ferrous ion & $\mathrm{Fe}^{2+}$ & $\mathrm{aq}$ & $-78.9 \mathrm{~kJ} \mathrm{~mol}^{-1}$ \\
\cline { 3 - 4 } Proton & $\mathrm{H}^{+}$ & $\mathrm{aq}$ & $0 \mathrm{~kJ} \mathrm{~mol}^{-1}$ \\
\cline { 3 - 4 } Carbon dioxide & $\mathrm{CO}_{2}$ & $\mathrm{aq}$ & $-386 \mathrm{~kJ} \mathrm{~mol}^{-1}$ \\
\cline { 3 - 4 } Ferric ion & $\mathrm{Fe}^{3+}$ & $\mathrm{aq}$ & $-4.6 \mathrm{~kJ} \mathrm{~mol}^{-1}$ \\
\cline { 3 - 4 } Water & $\mathrm{H}_{2} \mathrm{O}$ & $\mathrm{l}$ & $-237.2 \mathrm{~kJ} \mathrm{~mol}^{-1}$ \\
\cline { 3 - 4 } Methane & $\mathrm{CH}_{4}$ & $\mathrm{aq}$ & $-34.4 \mathrm{~kJ} \mathrm{~mol}^{-1}$ \\
\cline { 3 - 4 } Bicarbonate & $\mathrm{HCO}_{3}^{-}$ & $\mathrm{aq}$ & $-586.9 \mathrm{~kJ} \mathrm{~mol}^{-1}$ \\
\hline
\end{tabular}




\section{S4. Electronic Supplementary Databases}

S.DB.1| Microbial composition of Lake Kinneret sediments and slurry incubations based on SILVA (V132) database -Taxonomic classification (up until genus level) and abundance (in percentage) based on metagenomic reads to SILVA (V132) database. Eukaryote, Chloroplast, Mitochondria sequences were removed before normalization. https://doi.org/10.6084/m9.figshare.12933863.v1

S.DB.2| Microbial composition of Lake Kinneret sediments and slurry incubations based on MAR (MARine) database -Microbial abundance based on read mapping to MAR (MARine) database of prokaryotic genomes. https://doi.org/10.6084/m9.figshare.11800875.v3

S.DB.3| Abundance and classification of genes in Lake Kinneret sediments and slurry incubations based on KEGG orthology (in units of counts per million (CPM). https://doi.org/10.6084/m9.figshare.12933893.v1

S.DB.4| ANME2d Multiheme c-type cytochromes (MHC)sequences used as a query for BLASTing against Lake Kinneret sediment metagenome. https://doi.org/10.6084/m9.figshare.12933905.v1

S.DB.5 Abundance (in units of counts per million (CPM)) of genes encoding for F420:methanophenazine dehydrogenase complex (fpoABCDHIJKLMNO) in Lake Kinneret sediments and slurry incubations. https://doi.org/10.6084/m9.figshare.12933908.v1

S.DB.6 Metagenomic hits (amino acid sequences) for methanogenesis related enzymes -FwdC/FmdC, Ftr, Mch , MtrA, Mer, Mtd, McrA. https://doi.org/10.6084/m9.figshare.13091126.v2

S.DB.7| Metagenomic hits (amino acid sequences) for extracellular electron transfer related enzymes MHC, OmcS and PilA. https://doi.org/10.6084/m9.figshare.13092821.v1

S.DB.8| Metagenomic hits (amino acid sequences) for heterodisulfide reductase subunits A, D and E. https://doi.org/10.6084/m9.figshare.13092842.v1

\section{References}

Andersen, K. S., R. H. Kirkegaard, S. M. Karst, and M. Albertsen. 2018. ampvis2: an R package to analyse and visualise 16S rRNA amplicon data. bioRxiv 299537. doi:10.1101/299537

Bar-Or, I., M. Elvert, W. Eckert, A. Kushmaro, H. Vigderovich, Q. Zhu, E. Ben-Dov, and O. Sivan. 2017. Iron-coupled anaerobic oxidation of methane performed by a mixed bacterial-archaeal community based on poorly reactive minerals. Environ. Sci. Technol. 51: 12293-12301. doi:10.1021/acs.est.7b03126

Callahan, B. J., P. J. McMurdie, M. J. Rosen, A. W. Han, A. J. A. Johnson, and S. P. Holmes. 2016. DADA2: High-resolution sample inference from Illumina amplicon data. Nat. Methods 13: 581-583. doi:10.1038/nmeth.3869

Katoh, K., and D. M. Standley. 2013. MAFFT multiple sequence alignment software version 7: Improvements in performance and usability. Mol. Biol. Evol. 30: 772780. doi:10.1093/molbev/mst010

McMurdie, P. J., and S. Holmes. 2013. Phyloseq: An R package for reproducible interactive analysis and graphics of microbiome census data. PLoS One 8. doi:10.1371/journal.pone.0061217

Price, M. N., P. S. Dehal, and A. P. Arkin. 2009. Fasttree: Computing large minimum evolution trees with profiles instead of a distance matrix. Mol. Biol. Evol. 26: 1641-1650. doi:10.1093/molbev/msp077

R Core Team. 2018. R: A Language and Environment for Statistical Computing. 
Rideout, J. R., M. R. Dillon, N. A. Bokulich, and others. 2018. QIIME 2 :

Reproducible, interactive, scalable, and extensible microbiome data science. PeerJ Prepr. doi:10.7287/peerj.preprints.27295

Wickham, H. 2009. ggplot2: Elegant Graphics for Data Analysis, Springer-Verlag. 